\title{
Theoretical Investigation of the Black-body Zeeman Shift for Microwave Atomic Clocks
}

\author{
Jize $\operatorname{Han}^{1}$, Yani Zuo ${ }^{1}$, Jianwei Zhang ${ }^{2,3}$, a, and Lijun Wang ${ }^{1,2,3}$ \\ 1 Department of Physics, Tsinghua University, Beijing 100084 \\ 2 State Key Laboratory of Precision Measurement Technology and Instruments, Tsinghua University, Beijing 100084 \\ 3 Department of Precision Instruments, Tsinghua University, Beijing 100084
}

Received: date / Revised version: date

\begin{abstract}
With the development of microwave atomic clocks, the Zeeman shifts for the spectral lines of black-body radiation need to be investigated carefully. In this Letter, the frequency shifts of hyperfine splittings of atomic ground states due to the magnetic field of black-body radiation are reported. The relative frequency shifts of different alkali atoms and alkali-like ions, which could be candidates of microwave atomic clocks, were calculated. The results vary from $-0.977 \times 10^{-17}[T(K) / 300]^{2}$ to $-1.947 \times 10^{-17}[T(K) / 300]^{2}$ for different atoms considered. These results are consistent with previous work but with greater precision, detailed derivations, and a clear physical picture.
\end{abstract}

PACS. No more than four PACS codes should be provided

\section{Introduction}

For any atomic system, the energy levels are shifted by black-body radiation (BBR). These shifts may be ignored because their values are small. With the development of atomic clocks, they become one of the main components of frequency shifts, and indubitably have to be considered. Regarding microwave and optical atomic clocks, there are many theoretical ${ }^{[1-8]}$ and experimental $[9,10]$ studies of BBR shifts. However, most of those studies focus on BBR Stark (BBRS) shifts, because they are much larger than the BBR Zeeman (BBRZ) shifts pertinent for microwave atomic clocks. To the best of our knowledge, there are only a few theoretical studies ${ }^{[11-12]}$ on the BBRZ shifts and no experimental results. To date, the frequency uncertainty of the state-of-the-art cesium fountain has been decreased to order $1 \mathrm{E}-16$. It is worthwhile considering more carefully the BBRZ shifts for microwave atomic clocks as the uncertainties in frequency may improved to order $1 \mathrm{E}-17$ in the next decade.

In this Letter, we give a detailed derivation of the BBRZ shifts for the ground state of alkali atoms and alkali-like ions, which could be used in microwave atomic clocks. This theoretical investigation of the BBRZ shifts may be important for the development of microwave atomic clocks.

\section{Theory}

\subsection{Black-body radiation field}

According to Planck's radiation law,

$$
\begin{aligned}
\int_{0}^{+\infty} u(\omega) d \omega & =\int_{0}^{+\infty} \frac{\hbar}{\pi^{2} c^{3}} \frac{\omega^{3}}{e^{\frac{\hbar \omega}{k T}}-1} d w \\
& =\frac{1}{2} \varepsilon_{0}\left\langle E^{2}(t)\right\rangle+\frac{1}{2 \mu_{0}}\left\langle B^{2}(t)\right\rangle,
\end{aligned}
$$

where $u(\omega) d \omega$ is the field energy density in the bandwidth $d \omega$ around angular frequency $\omega,\left\langle E^{2}(t)\right\rangle$ and $\left\langle B^{2}(t)\right\rangle$ are the mean-squares of the electric and magnetic fields, respectively. The mean-squares of the fields are defined as

$$
\left\langle B^{2}(t)\right\rangle=\frac{1}{2} \int_{0}^{+\infty} B^{2}(\omega) d \omega
$$

and

$$
\left\langle E^{2}(t)\right\rangle=\frac{1}{2} \int_{0}^{+\infty} E^{2}(\omega) d \omega .
$$

Here, $E^{2}(\omega)$ and $B^{2}(\omega)$ are the field energy densities in a bandwidth $d \omega$ around $\omega$ of the electric and magnetic fields, respectively.

According to the theory of electromagnetism, the fields in an electromagnetic wave have the same energy. Thus, $B^{2}(\omega) d \omega$ is

$$
B^{2}(\omega) d \omega=\frac{2 \mu_{0} \hbar}{\pi^{2} c^{3}} \frac{\omega^{3}}{e^{\frac{\hbar \omega}{k T}}-1} d w
$$




\subsection{Radiation field interacting with two-level atoms}

We consider a simple situation where a magnetic field oscillates at angular frequency $\omega$ interacting with two-level atoms. The evolution of the system is governed by the time-dependent Schrödinger equation

$$
i \hbar \frac{d \Psi}{d t}=\widehat{H} \Psi
$$

with Hamiltonian written as

$$
\widehat{H}=\widehat{H}_{A}+\widehat{H}_{A F}(t),
$$

where

$$
\widehat{H}_{A F}(t)=\boldsymbol{\mu} \cdot \boldsymbol{B} \cos \omega t .
$$

Here, $\widehat{H}_{A}$ is the Hamiltonian of the free atom, $\widehat{H}_{A F}$ the atom-field interaction Hamiltonian, $\boldsymbol{\mu}$ the magnetic moment, and $\boldsymbol{B}$ the amplitude of the monochromatic field.

The wave function associated with $\widehat{H}$ is a superposition of the two eigenfunctions,

$$
\Psi(r, t)=C_{g}(t) \Psi_{g}(r) e^{-i \omega_{g} t}+C_{e}(t) \Psi_{e}(r) e^{-i \omega_{e} t},
$$

where $\omega_{g}$ and $\omega_{e}$ are the frequencies of the ground and excited state, and $C_{g}$ and $C_{e}$ the coefficients of overlap for the two states, respectively.

We may next derive two coupled equations for these coefficients,

$$
\left\{\begin{array}{c}
i \dot{C}_{g}=C_{e}\left[e^{i\left(\omega-\omega_{0}\right) t}+e^{-i\left(\omega+\omega_{0}\right) t}\right] \frac{\Omega}{2} \\
i \dot{C}_{e}=C_{g}\left[e^{-i\left(\omega-\omega_{0}\right) t}+e^{i\left(\omega+\omega_{0}\right) t}\right] \frac{\Omega}{2},
\end{array}\right.
$$

where $\Omega=\langle g|\boldsymbol{\mu} \cdot \boldsymbol{B}| e\rangle / \hbar$ is the magnetic Rabi frequency, and $\omega_{0}=\omega_{e}-\omega_{g}$ is the resonance frequency between the two levels.

If the frequency of the radiation field $\omega$ is near resonance, $\left|\omega-\omega_{0}\right| \ll \omega_{0}$, we use the rotating-wave approximation (RWA) to simplify Eq. (9), and we have

$$
\left\{\begin{array}{l}
i \dot{C}_{g}=C_{e} e^{i\left(\omega-\omega_{0}\right) t} \frac{\Omega}{2} \\
i \dot{C}_{e}=C_{g} e^{-i\left(\omega-\omega_{0}\right) t} \frac{\Omega}{2}
\end{array}\right.
$$

After solving the secular equations, Eq. (10), the frequency shifts induced by the rotating term is ${ }^{[13]}$

$$
\begin{aligned}
\Delta \omega_{\text {rot }} & =-\frac{\left(b_{e}^{2}+b_{g}^{2}\right) \mu_{B}^{2} B^{2}}{4\left(\omega-\omega_{0}\right)} \\
& =-\frac{b^{2} \mu_{B}^{2} B^{2}}{4\left(\omega-\omega_{0}\right)},
\end{aligned}
$$

where $b=|\langle g|\boldsymbol{\mu}| e\rangle| / \mu_{B}$ is the coupling coefficient, $b_{g}$ and $b_{e}$ are the coupling coefficients of the ground and excited states, respectively, and the total coupling coefficient $b=$ $\sqrt{b_{e}^{2}-\left(-b_{g}^{2}\right)}$
For far detuning, $\left|\omega-\omega_{0}\right| \approx \omega_{0}$ or $\omega$, applying the RWA is not valid. Instead, the counter-rotating terms are given by

$$
\left\{\begin{array}{l}
i \dot{C}_{g}=C_{e} e^{-i\left(\omega+\omega_{0}\right) t} \frac{\Omega}{2} \\
i \dot{C}_{e}=C_{g} e^{i\left(\omega+\omega_{0}\right) t} \frac{\Omega}{2}
\end{array}\right.
$$

We may treat the counter-rotating terms as another monochromatic field with frequency $-\omega$. The frequency shift has the same form except $\omega$ is replaced by $-\omega$. Thus, the frequency shift caused by the counter-rotating term is $[14]$

$$
\Delta \omega_{c r o t}=\frac{b^{2} \mu_{B}^{2} B^{2}}{4\left(\omega+\omega_{0}\right) \hbar^{2}} .
$$

Combining Eqs. (11) and (13), the total frequency shift of the far-detuning situation are

$$
\begin{aligned}
\Delta \omega_{f d e t} & =\Delta \omega_{\text {rot }}+\Delta \omega_{\text {crot }} \\
& =-\frac{\omega_{0} b^{2} \mu_{B}^{2} B^{2}}{2\left(\omega^{2}-\omega_{0}^{2}\right) \hbar^{2}} .
\end{aligned}
$$

For near resonance, there is a more accurate expression for the frequency shift ${ }^{[15,16]}$. Therefore, the complete expression of the ground state frequency shift for the two distinct situations is

$$
\Delta \omega=\left\{\begin{array}{l}
-\frac{\omega_{0} b^{2} \mu_{B}^{2} B^{2}(\omega)}{2\left(\omega^{2}-\omega_{0}^{2}\right) \hbar^{2}} \\
\omega \in\left[0, \omega_{0}-\delta \omega_{0}\right] \cup\left[\omega_{0}+\delta \omega_{0}, \infty\right] \\
-\frac{b^{2} \mu_{B}^{2} B^{2}(\omega)}{2 \hbar^{2}} \cdot \frac{\omega-\omega_{0}}{\left(\omega-\omega_{0}\right)^{2}+\frac{b^{2} \mu_{B}^{2} B^{2}(\omega)}{\hbar^{2}}}, \\
\omega \in\left[\omega_{0}-\delta \omega_{0}, \omega_{0}+\delta \omega_{0}\right]
\end{array}\right.
$$

with the excited state having the opposite sign. In Eq. (15), we choose $\omega_{0} \mp \delta \omega_{0}$ as the lower and upper limits of the near-resonance situation. Without loss of generality, we set $\delta \omega_{0}=0.01 \omega_{0}$ for the calculation.

\subsection{Calculation of the coupling coefficients}

To calculate $\Delta \omega$, we need to calculate the coupling coefficient,

$$
b=\frac{|\langle g|\boldsymbol{\mu}| e\rangle|}{\mu_{B}}=\frac{\left|\left\langle\boldsymbol{F} m_{F}|\boldsymbol{\mu}| \boldsymbol{F}^{\prime} m_{F}^{\prime}\right\rangle\right|}{\mu_{B}},
$$

between the ground state and the excited state. Because the total angular momentum $\boldsymbol{F}$ and its magnetic quantum number $m_{F}$ are different for $g$ and $e$, we cannot obtain the coupling coefficient $b$ directly using the coupling formula for the total electronic angular momentum g-factor $g_{J}$, and nuclear spin angular momentum g-factor $g_{I}$. Instead, we need to transform the coupled representation into an 


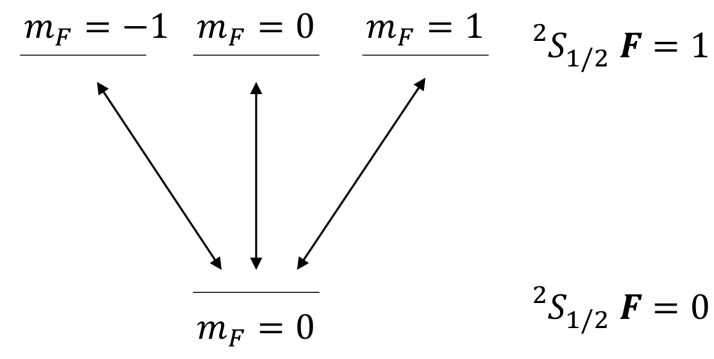

Fig. 1. Energy levels for the ground state of $\boldsymbol{I}=1 / 2$ atom

$$
\begin{aligned}
& |e\rangle-\left|\frac{1}{2} \frac{1}{2} ; 11\right|=\left|\frac{1}{2} \frac{1}{2} ; \frac{1}{2} \frac{1}{2}\right| \\
& |g\rangle \longrightarrow\left|\frac{1}{2} \frac{1}{2} ; 00\right|=\frac{1}{\sqrt{2}}\left|\frac{1}{2} \frac{1}{2} ; \frac{1}{2}-\frac{1}{2}\right|-\frac{1}{\sqrt{2}}\left|\frac{1}{2}-\frac{1}{2} ; \frac{1}{2} \frac{1}{2}\right|
\end{aligned}
$$

Fig. 2. Uncoupled representations of $|00\rangle|11\rangle$

uncoupled representation using the Clebsch-Gordan coefficients,

$$
\begin{aligned}
& \left|\boldsymbol{J} \boldsymbol{I} ; \boldsymbol{F} m_{F}\right\rangle= \\
& \sum_{m_{J}=-J}^{J} \sum_{m_{I}=-I}^{I}\left|\boldsymbol{J} m_{J} ; \boldsymbol{I} m_{I}\right\rangle\left\langle\boldsymbol{J} m_{J} ; \boldsymbol{I} m_{I} \mid \boldsymbol{J} \boldsymbol{I} ; \boldsymbol{F} m_{F}\right\rangle .
\end{aligned}
$$

For example, the clock transition for atoms with nuclear spin $\boldsymbol{I}=1 / 2$ is the hyperfine splittings ${ }^{2} S_{1 / 2} \boldsymbol{F}=0$, $m_{F}=0$ to ${ }^{2} S_{1 / 2} \boldsymbol{F}=1, m_{F}=0$; the energy levels are shown in Fig. 1 .

As shown in Fig. 1, we need to consider all other states that could influence our target states. In this instance, there are three transitions that shift the ground state level of the clock transition: $|00| \rightarrow|11\rangle,|00\rangle \rightarrow|10\rangle$, and $|00\rangle \rightarrow|1-1\rangle$.

For the energy transition $|00\rangle \rightarrow|11\rangle$, the uncoupled representations of each levels are shown in Fig. 2.

Thus, the coupling coefficient $b_{g}$ is

$$
\begin{aligned}
b_{g}= & \left\langle 00\left|g_{J} \boldsymbol{J}+g_{I} \boldsymbol{I}\right| 11\right\rangle \\
= & \left(\frac{1}{\sqrt{2}}\left\langle\frac{1}{2} \frac{1}{2} ; \frac{1}{2}-\frac{1}{2}\right|-\frac{1}{\sqrt{2}}\left\langle\frac{1}{2}-\frac{1}{2} ; \frac{1}{2} \frac{1}{2}\right|\right) \\
& \cdot\left(g_{J} \boldsymbol{J}+g_{I} \boldsymbol{I}\right)\left(\left|\frac{1}{2} \frac{1}{2} ; \frac{1}{2} \frac{1}{2}\right\rangle\right) \\
= & \frac{1}{2 \sqrt{2}}\left(g_{J}-g_{I}\right) .
\end{aligned}
$$

\begin{tabular}{|c|c|c|}
\hline $\begin{array}{l}\text { Nuclear } \\
\text { spin }\end{array}$ & $\begin{array}{l}\text { Alkali atoms and } \\
\text { Alkali-like ions }\end{array}$ & $\begin{array}{l}\text { Total Coupling } \\
\text { Coefficient } b\end{array}$ \\
\hline$\frac{1}{2}$ & $\begin{array}{l}{ }^{1} \mathrm{H},{ }^{3} \mathrm{H},{ }^{3} \mathrm{He}^{+},{ }^{111} \mathrm{Cd}^{+} \\
{ }^{113} \mathrm{Cd}^{+},{ }^{171} \mathrm{Yb}^{+},{ }^{199} \mathrm{Hg}^{+}\end{array}$ & $\frac{\sqrt{3}}{2}\left(g_{J}-g_{I}\right)$ \\
\hline$\frac{3}{2}$ & $\begin{array}{l}{ }^{7} \mathrm{Li},{ }^{9} \mathrm{Be}^{+},{ }^{39} \mathrm{~K},{ }^{41} \mathrm{~K},{ }^{201} \mathrm{Hg}^{+} \\
{ }^{23} \mathrm{Na},{ }^{87} \mathrm{Rb},{ }^{135} \mathrm{Ba}^{+},{ }^{137} \mathrm{Ba}^{+}\end{array}$ & $\begin{array}{l}\frac{1}{\sqrt{3}}\left\{\left[(3+\sqrt{2}) g_{J}^{2}\right.\right. \\
\left.\left.-\frac{7 g_{J} g_{I}}{6}+\frac{3 g_{I}^{2}}{4}\right]\right\}^{\frac{1}{2}}\end{array}$ \\
\hline$\frac{5}{2}$ & ${ }^{25} \mathrm{Mg}^{+},{ }^{67} \mathrm{Zn}^{+},{ }^{85} \mathrm{Rb},{ }^{173} \mathrm{Yb}^{+}$ & $\begin{array}{l}\frac{1}{\sqrt{3}}\left\{\left[(3+\sqrt{2}) g_{J}^{2}\right.\right. \\
\left.\left.-\frac{7 g_{J} g_{I}}{6}+\frac{3 g_{I}^{2}}{4}\right]\right\}^{\frac{1}{2}}\end{array}$ \\
\hline$\frac{7}{2}$ & ${ }^{43} \mathrm{Ca}^{+},{ }^{133} \mathrm{Cs}$ & $\begin{array}{l}\frac{1}{2 \sqrt{2}}\left\{\left[(8+\sqrt{15}) g_{J}^{2}\right.\right. \\
\left.\left.-9 g_{J} g_{I}+6 g_{I}^{2}\right]\right\}^{\frac{1}{2}}\end{array}$ \\
\hline$\frac{9}{2}$ & ${ }^{87} \mathrm{Sr}^{+}$ & $\begin{array}{l}\frac{1}{2 \sqrt{5}}\left\{\left[(5+\sqrt{6}) g_{J}^{2}\right.\right. \\
\left.\left.-22 g_{J} g_{I}+15 g_{I}^{2}\right]\right\}^{\frac{1}{2}}\end{array}$ \\
\hline
\end{tabular}

Using the same methods, we determined the coupling coefficients for $|00\rangle \rightarrow|10\rangle$ and $|00\rangle \rightarrow|1-1\rangle$. The results of $b_{g}$ are $\left(g_{J}-g_{I}\right) / 2$ and $\left(g_{J}-g_{I}\right) / 2 \sqrt{2}$, respectively.

Only the $|10\rangle \rightarrow|00\rangle$ transition shifts the excited state of the clock transition; specifically, we find $b_{e}$ is $\left(g_{J}-g_{I}\right) /$ 2 .
Table 1. Coupling coefficients for different nuclear spins

The total coupling coefficient of the clock transition with $\boldsymbol{I}=1 / 2$ is

$$
b=\sqrt{b_{e}^{2}-\left(-b_{g}^{2}\right)}=\frac{\sqrt{3}}{2}\left(g_{J}-g_{I}\right) .
$$

For different nuclear spins, the coupling coefficients are calculated in the same way; the results are listed in Table 1 .

\section{Results and discussion}

The BBR field is a broad-band radiation field with a uniform spatial distribution. There is only $1 / 3$ of total radiation field parallel to the direction of the static magnetic field, which induces the $m_{F}=0 \rightarrow m_{F}=0$ transition. The remaining part of the radiation field is orthogonal to the static magnetic field, and may decompose into left and right circular-polarized light, which induces the $m_{F}=0 \rightarrow m_{F}= \pm 1$ transitions ${ }^{[17]}$. To obtain the total BBRZ shifts, Eq. (15) is integrated for all $\omega$, and multiplied by $1 / 3$ to account for the spatial distribution,

$$
\begin{aligned}
\frac{\Delta \omega}{\omega_{0}}= & -\int_{0}^{\omega_{0}-\delta \omega_{0}} \frac{b^{2} \mu_{B}^{2} B^{2}(\omega)}{6 \hbar^{2}\left(\omega^{2}-\omega_{0}^{2}\right)} d w \\
& -\int_{\omega_{0}-\delta \omega_{0}}^{\omega_{0}+\delta \omega_{0}} \frac{b^{2} \mu_{B}^{2} B^{2}(\omega)}{6 \omega_{0} \hbar^{2}} \cdot \frac{\omega-\omega_{0}}{\left(\omega-\omega_{0}\right)^{2}+\frac{b^{2} \mu_{B}^{2} B^{2}(\omega)}{\hbar^{2}}} d w \\
& -\int_{\omega_{0}+\delta \omega_{0}}^{+\infty} \frac{b^{2} \mu_{B}^{2} B^{2}(\omega)}{6 \hbar^{2}\left(\omega^{2}-\omega_{0}^{2}\right)} d w .
\end{aligned}
$$

Using Eq. (20), we then calculated the frequency shifts for each candidate atom of a microwave atomic clock. The data required concerning the atomic structure are cited from Refs. [18-21]. The results are listed in Table 2.

The above-described procedure used for the evaluation does not include the influence of the fine structure. 
Table 2. Relative BBRZ shifts for the ground states of alkali atoms and alkali-like ions

\begin{tabular}{|c|c|c|}
\hline $\begin{array}{l}\text { Nuclear } \\
\text { spin } \boldsymbol{I}\end{array}$ & $\begin{array}{c}\text { Alkali atoms and } \\
\text { Alkali-like ions }\end{array}$ & $\begin{array}{l}\text { Relative BBRZ Frequency } \\
\text { shifts } \frac{\Delta \omega}{\omega_{0}}\left[\frac{T(K)}{300}\right]^{2}\left(\times 10^{-17}\right)\end{array}$ \\
\hline \multirow{7}{*}{$\frac{1}{2}$} & ${ }^{1} \mathrm{H}$ & -0.977 \\
\hline & ${ }^{3} \mathrm{H}$ & -0.977 \\
\hline & ${ }^{3} \mathrm{He}^{+}$ & -0.982 \\
\hline & ${ }^{111} \mathrm{Cd}^{+}$ & -0.981 \\
\hline & ${ }^{113} \mathrm{Cd}^{+}$ & -0.981 \\
\hline & ${ }^{171} \mathrm{Yb}^{+}$ & -0.980 \\
\hline & ${ }^{199} \mathrm{Hg}^{+}$ & -0.979 \\
\hline \multirow{9}{*}{$\frac{3}{2}$} & ${ }^{7} \mathrm{Li}$ & -1.872 \\
\hline & ${ }^{23} \mathrm{Na}$ & -1.872 \\
\hline & ${ }^{39} \mathrm{~K}$ & -1.873 \\
\hline & ${ }^{41} \mathrm{~K}$ & -1.873 \\
\hline & ${ }^{87} \mathrm{Rb}$ & -1.872 \\
\hline & ${ }^{9} \mathrm{Be}^{+}$ & -1.873 \\
\hline & ${ }^{135} \mathrm{Ba}^{+}$ & -1.872 \\
\hline & ${ }^{137} \mathrm{Ba}^{+}$ & -1.872 \\
\hline & ${ }^{201} \mathrm{Hg}^{+}$ & -1.874 \\
\hline \multirow{4}{*}{$\frac{5}{2}$} & ${ }^{85} \mathrm{Rb}$ & -1.923 \\
\hline & ${ }^{25} \mathrm{Mg}^{+}$ & -1.923 \\
\hline & ${ }^{67} \mathrm{Zn}^{+}$ & -1.922 \\
\hline & ${ }^{173} \mathrm{Yb}^{+}$ & -1.922 \\
\hline \multirow{2}{*}{$\frac{7}{2}$} & ${ }^{133} \mathrm{Cs}$ & -1.940 \\
\hline & ${ }^{43} \mathrm{Ca}^{+}$ & -1.939 \\
\hline$\frac{9}{2}$ & ${ }^{87} \mathrm{Sr}^{+}$ & -1.947 \\
\hline
\end{tabular}

Because the BBRZ shift is a magnetic dipole interaction, the energy shift due to the hyperfine structure is the dominating term. We see these results using second-order perturbation theory,

$$
\Delta E \approx \sum_{e \neq g} \frac{\left|\left\langle e\left|\widehat{H}_{A F}\right| g\right\rangle\right|^{2}}{E(e)-E(g)},
$$

where $\widehat{H}_{A F}$ is the atom-field interaction Hamiltonian, $e$ the excited state, and $g$ the ground state. The hyperfine splittings are usually several gigahertz, but the energy difference of the fine structure is of order $100 \mathrm{THz}$. In the present circumstance, the coupling of other fine-structure levels can be neglected.

Itano and coworkers ${ }^{[11]}$ analyzed the BBRZ shifts for any alkali atoms and alkali-like ions in the ground state, and presented a result of $-1.034 \times 10^{-17}[T(K) / 300]^{2}$. Our results are fairly close to their result. In addition, we derived the BBRZ shifts for different atoms with different nuclear spins and different hyperfine splittings, and they may give some guidance in choosing atomic species for microwave atomic clocks.

In Ref. [12], the BBRZ shifts of cesium were calculated to be $-4.933 \times 10^{-15}[T(K) / 300]^{3}$. Their results are quite different from Itano and coworkers and from our results. The key problem of Ref. [12] may have been the failure to take into account the counter-rotating terms when using the RWA .

In conclusion, this Letter gives a simple and detailed derivation of the BBRZ shifts for the ground states of alkali atoms and alkali-like ions through two-level system analysis. The calculation results consistent with previous works very well. The analytical results might have been very useful for experimental physicist doing microwave atomic clocks to estimate BBR Zeeman shifts and the further improvement of microwave atomic clocks.

\section{Acknowledgment}

This project is supported by National Key Research and Development Program of China (No.2016YFA0302101), and the Initiative program of State Key Laboratory of Precision Measurement Technology and Instruments.

We thank S.G.Porsev and Y.M.Yu for helpful discussions and suggestions.

\section{References}

1. S. G. Porsev, A. Derevianko, Phys. Rev. A 74, 020502(R) (2006)

2. D. Jiang et al., J. Phys. B: At. Mol. Opt. Phys. 42, 154020 (2009)

3. K. Beloy et al., Phys. Rev. Lett. 97, 040801 (2006)

4. B. Arora, M. S. Safronova, C. W. Clark, Phys. Rev. A 76, 064501 (2007)

5. M. S. Safronova et al., Phys. Rev. A 87, 012509 (2013)

6. M. S. Safronova, U. I. Safronova, Phys. Rev. A 83, 012503 (2011)

7. S. Micalizio et al., Phys. Rev. A 69, 053401 (2004)

8. U. I. Safronova, M. S. Safronova. Phys. Rev. A 79, 022512 (2009)

9. T. Middelmann et al., Phys. Rev. Lett. 109, 263004 (2012)

10. M. S. Safronova et al., Phys. Rev. A 87, 012509 (2013)

11. W. M. Itano, L. L. Lewis, D. J. Wineland, Phys. Rev. A 25, 1233(R) (1982)

12. W. Gao, X. Ke, H. Liu, Acta Optica Sinica 20, 3 (2000), (in Chinese)

13. C. J. Foot, Atomic physics (Oxford University Press, 2005) 14. D. A. Steck, Quantum and Atom Optics (available online at http://steck.us/teaching (revision 0.12.0, 16 May 2017))

15. A. Xing, T. Dong, X. Huang, Acta Sinica Quantum Optica 7, 1, (2001) (in Chinese)

16. J. B. Chen et al., Chin. Phys. Lett 18, 2 (2001)

17. P. Ewart, Atomic Physics Lecture notes (Department of physics, University of Oxford)

18. J. Vanier, C. Audoin, Quantum Physics of Atomic Frequency Standards Volume 1 (CRC Press, 1989) 
19. G. Dixit et al., J. Phys. B: At. Mol. Opt. Phys 41, 025001

(2008)

20. J. Benhelm et al., Phys. Rev. A 75032506 (2007)

21. H. Sunaoshi et al., Hyperfine Interactions 78 (1993) 